\title{
Quasibiennial Oscillations of the North-South Asymmetry
}

\author{
O. G. Badalyan'1, V. N. Obridko', J. Rybák², and J. Sýkora² \\ ${ }^{1}$ Institute of Terrestrial Magnetism, Ionosphere, and Radiowave Propagation, Russian Academy of Sciences, \\ Troitsk, Moscow oblast, 142190 Russia \\ ${ }^{2}$ Astronomical Institute, Slovak Academy of Sciences, Tatranská Lomnica, 05960 Slovak Republic
}

Received December 25, 2004; in final form, February 17, 2005

\begin{abstract}
The north-south (N-S) asymmetry of the solar activity $(A)$, which reflects differences in the behavior of the northern and southern hemispheres of the Sun, is studied using data on the brightness of the coronal green line, the total number and area of sunspots, and the net magnetic flux. The spatial and temporal distributions and correlations between the $A$ values represented by these indices are considered. The characteristic time variations in $A$ are similar for all the indices, on both long and short time scales. Quasibiennial oscillations (QBOs) can be traced in the asymmetries of all four indices. A detailed study of the QBOs is carried out based on spectral-variation and wavelet analyses. Long-term increases and decreases occur synchronously in the asymmetries of various indices and are much more pronounced in $A$ than in the indices themselves. A negative correlation between the power of the QBOs and the asymmetry of $A$ can be traced; it is most clearly manifest as a substantial diminishing of the QBOs during the mid-1960s, which coincided with an especially strong increase in $A$. Our analysis shows that the $\mathrm{N}-\mathrm{S}$ asymmetry is probably a fundamental property that controls the coupling and degree of coincidence between the magnetic-field-generation mechanisms operating in the northern and southern hemispheres.

(c) 2005 Pleiades Publishing, Inc.
\end{abstract}

\section{INTRODUCTION}

Over the long period in which solar activity has been investigated, the Sun has usually been treated as a single object, without division into hemispheres. It has been assumed that solar processes occur in the same manner in the northern and southern hemispheres. Numerous solar-activity indices have been introduced for the Sun as a whole-the Wolf number, the number of polar faculae, the full radio flux, etc. This approach made it possible to reveal the basic properties of the solar cycle. Nearly synchronous cyclic variations were detected in the behavior of various indices reflecting manifestations of activity at all levels in the solar atmosphere, from the photosphere to corona. Similar cyclic variations were also traced in interplanetary space and in geomagneticactivity indices. Various theoretical concepts, such as the theory of the differential rotation of the Sun, the dynamo theory, and helioseismology, were developed assuming that both solar hemispheres were identical.

However, it gradually became clear that the northern and southern solar hemispheres do not display quite the same behavior. The time variations in various solar-activity indices demonstrate phase and power mismatches between the two hemispheres over intervals of several months to several years. These differences are manifest in numerous solar-activity indices and are commonly described as the northsouth $(\mathrm{N}-\mathrm{S})$ asymmetry. The differences represented by the $\mathrm{N}-\mathrm{S}$ asymmetry are small compared to the indices themselves. It is noteworthy that, for a fairly long time, many investigators were inclined to consider this asymmetry an artifact resulting from observational errors and statistically insignificant fluctuations of measurable quantities. For this reason, even the recent study [1] opened its list of basic conclusions with the statement that the $\mathrm{N}-\mathrm{S}$ asymmetry of sunspot areas is indeed statistically meaningful and represents a real phenomenon.

Although the asymmetry has been studied for a considerable time, its nature remains unclear, and the identification of new observational facts reflecting various aspects of this interesting phenomenon is still in progress. The sunspot asymmetry has been studied most thoroughly, using sunspot areas and some other sunspot indices over various time intervals (see, e.g., [1-3]. The asymmetries of many other solaractivity indices describing filaments, prominences, unsteady solar phenomena, and geomagnetic activity have also been analyzed. Knaack et al. [4] recently considered asymmetry in the solar magnetic field. A fairly detailed review of studies of the asymmetries in various indices can be found in $[1,3,5,6]$.

A number of characteristic features of the asymmetry have been revealed. In particular, a number 
of studies have noted that the northern hemisphere was much more active over a long period during the second half of the 20th century. Quasiperiodic oscillations in the wide range of frequencies were detected in the asymmetries of various solar-activity indices (see, e.g., $[1,4,7,8])$. Furthermore, due to the presence of this asymmetry, the active longitudes do not coincide in the northern and southern hemispheres, and observations even suggest that they are antipodal [9, p. 264].

Some studies have compared the asymmetries in different solar-activity indices [2, 10-12]. These results suggest that the asymmetry behaves similarly in various indices and on various temporal and spatial scales. One of our areas of study is the verification and investigation of this hypothesis.

We consider here the asymmetries in four different solar-activity indices over a relatively long time interval. We have chosen the brightness of the coronal green line $I$, the total sunspot area $S_{p}$, the total sunspot number $Q$, and the net magnetic flux $\Phi$ for this study.

\section{We consider}

(1) the spatial and temporal distributions of the $\mathrm{N}-\mathrm{S}$ asymmetries for various solar-activity indices and correlations between these asymmetries on both short and long time scales;

(2) QBOs in the asymmetry of the activity indices and the spatial and temporal distributions of these QBOs; comparisons of the QBOs in the asymmetries with the QBOs in the activity indices themselves;

(3) the relationship between the power of QBOs in the asymmetry and the magnitude of asymmetry [itself].

Our results show that the $\mathrm{N}-\mathrm{S}$ asymmetry is a very significant and informative parameter of solar activity. The Conclusion presents a discussion of problems related to this phenomenon.

\section{CHARACTERIZATION OF THE CHOSEN ACTIVITY INDICES AND DATABASES}

An important property common to the chosen indices is that they all describe the activity of the Sun at a specific point on its surface. Most other indices are either integrated and so related to the entire Sun (the total flux of solar radiation, the total radio flux, etc.) or characterize nonstationary processes (the number, intensity, and coordinates of solar flares and coronal mass ejections), which are beyond the scope of our consideration. Digitized filtergrams of the solar disk are not readily available, and high-resolution radio maps have become available only fairly recently. Another important property of the indices we have chosen is that the corresponding series of observational data are long. The database of coronal green-line brightness measurements encompasses more than 5.5 activity cycles. Data on sunspot parameters and numbers are also available for this same period. Information on the total magnetic flux reduced to a uniform system is available starting from 1975 and so covers 2.5 cycles.

The brightness of the FeXIV $\lambda$ 530.3-nm coronal green line, $I$, is a very convenient index that can be used to study solar activity and its asymmetry in the corona. An important advantage of the index $I$ is that it can be determined virtually simultaneously for all heliographic latitudes. This index provides a uniform and contiguous digital field for all points of the disk over a long time interval based on regular observations. In this respect, it stands out from, for example, the Wolf numbers and sunspot areas (which emerge at low latitudes only) or the numbers of polar faculae (which reflect solar activity at high latitudes). The database used contains green-line intensities for each day in steps of $5^{\circ}$ in position angle, referenced to a $60^{\prime \prime}$ height over the limb.

Sunspots can be used to study the solar activity at the photospheric level. The advantage of sunspot indices is their relatively long data series, which appreciably overlaps with the time covered by green-line observations. Naturally, these indices can be used to study the activity of the Sun and its asymmetry in the equatorial zone only, at latitudes of up to $\sim 30^{\circ}$. We consider here two indices characterizing the sunspot activity the total area $S_{p}$ and total number $Q$ of the sunspots. We emphasize that we used the total number of sunspots rather than the traditional Wolf number. As will become clear below, the total sunspot number is an independent and very interesting index, which, in many cases, correlates better with the green-line asymmetry parameters than, for example, the sunspot areas or Wolf numbers. As follows from the studies of Kopecký and Kuklin (see the monograph [9] and references therein), the indices $S_{p}$ and $Q$ are related to the primary sunspot-formation indices in different ways.

Like the green-line brightness $I$, the magnetic flux $\Phi$ can, in principle, be determined for every point of the solar surface. In contrast to the sunspotactivity indices, this characteristic of the magnetic field is a continuous quantity. The magnetic flux is the main characteristic of the solar magnetic field, which determines all elements of solar activity, including the coronal structure and sunspot formation.

We used the following databases in our study.

(1) The brightness of the $\lambda 530.3-\mathrm{nm}$ coronal green line. This database contains observations obtained at several coronal stations and currently covers the period 1939-2001. The data of individual stations 

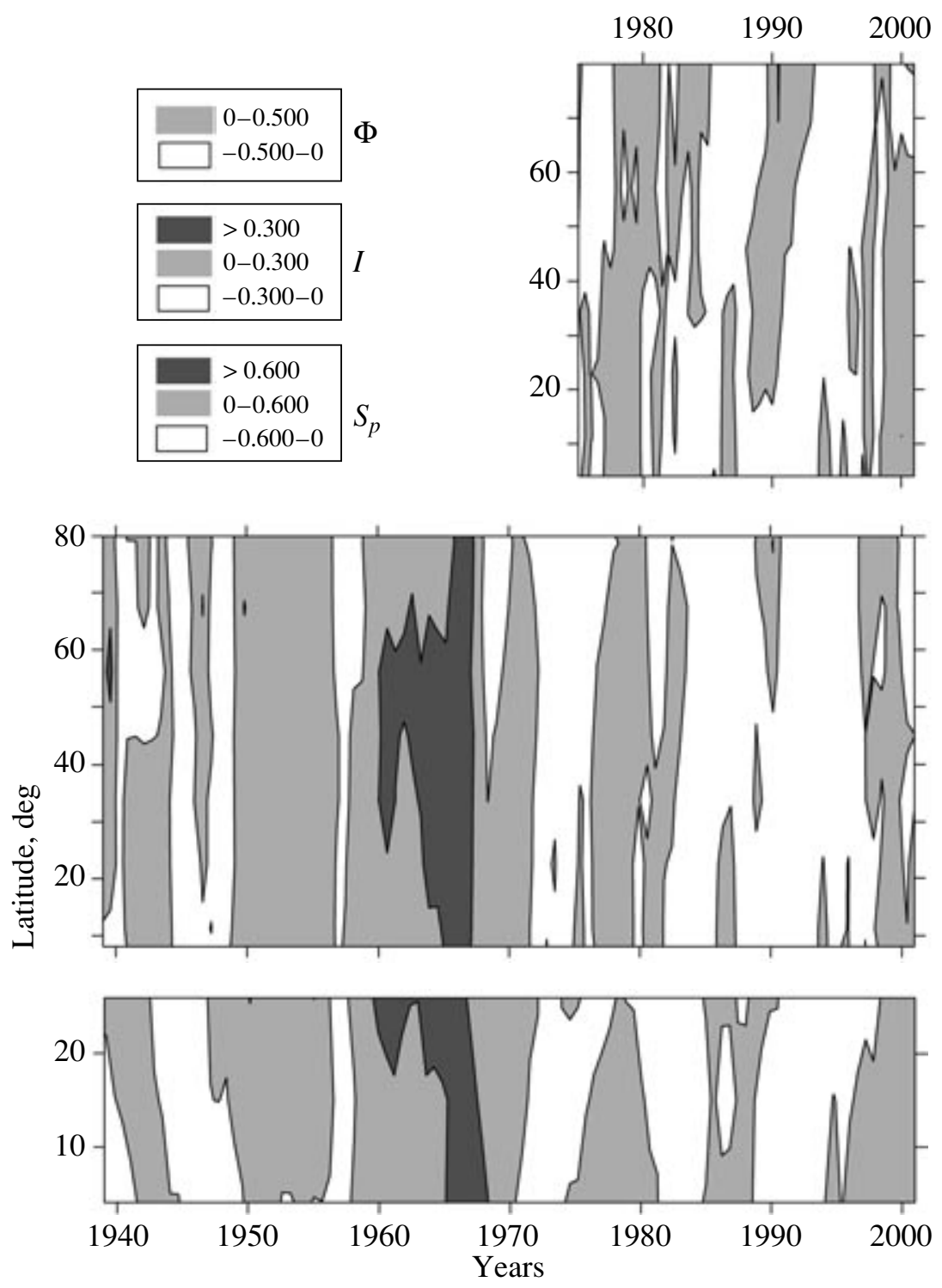

Fig. 1. Latitude-time diagram for the asymmetries in the net magnetic flux, coronal green-line brightness, and total sunspot area (from top to bottom). The gray scales for the maps are shown at the top left.

are reduced to a single photometric system; the technique used to unify the data is described in [1315], and a description of the database is also given in $[16,17]$.

(2) The total sunspot area. The monthly averages were calculated from the data of the Greenwich Observatory obtained via the Internet.

(3) The total sunspot number was also calculated from data of the Greenwich Observatory available via the Internet.

(4) The net magnetic flux. Data on the magnetic flux at the photospheric level are obtained from magnetic-field observations carried out at the Kitt Peak Observatory,

(ftp://argo.tuc.noao.edu/kpvt/synoptic/mag/), in the form of synoptic magnetic-field maps, which cover the interval 1975-2001,

(ftp://nsokp.nso.edu/kpvt/synoptic/).

Direct measurements yield the line-of-sight component of the magnetic field. Based on the assumption that the photospheric magnetic field is radial with respect to the solar surface, the observed values are divided by the cosine of the heliographic latitude. We used here the mean magnetic-flux values for each Carrington rotation and each $10^{\circ}$ latitude zone.

Thus, the activity indices whose asymmetry we consider correspond to different manifestations of solar activity. Unified statistical-analysis techniques were applied to all these data. This makes it possible to compare the results obtained for objects resulting 
Table 1. Correlation coefficients for the asymmetries of various pairs of indices at latitudes of $0^{\circ}-30^{\circ}$

\begin{tabular}{l|c}
\hline Indices & $k_{A}(X, Y)$ \\
\hline$S_{p}-Q$ & $0.916 \pm 0.006$ \\
$S_{p}-I$ & $0.800 \pm 0.013$ \\
$S_{p}-\Phi$ & $0.768 \pm 0.024$ \\
$Q-I$ & $0.860 \pm 0.010$ \\
$Q-\Phi$ & $0.800 \pm 0.021$ \\
$I-\Phi$ & $0.848 \pm 0.016$ \\
\hline
\end{tabular}

from completely different magnetic field-matter interactions. Note that we did not include nonstationary processes in our analysis.

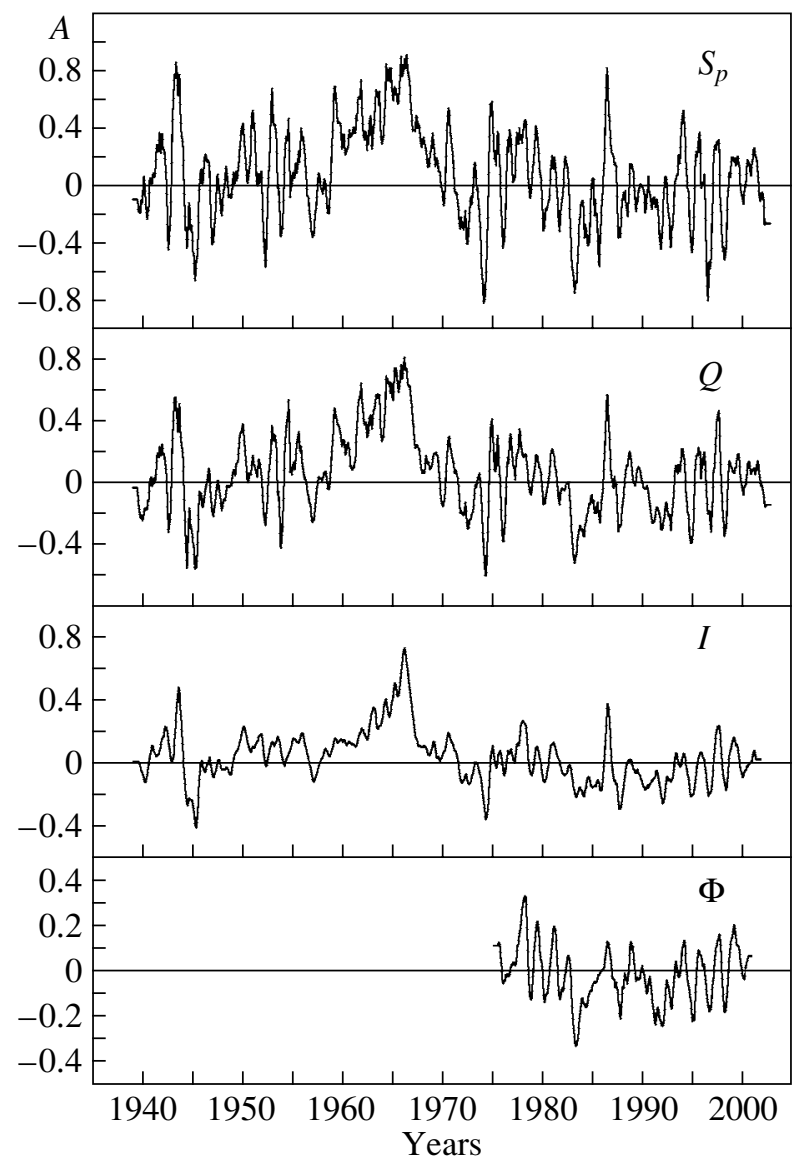

Fig. 2. Variation of the asymmetries in the four solaractivity indices in the zone of spot formation, $0^{\circ}-30^{\circ}$. Smoothing based on a 13th-order polynomial was done using the Savitzky-Golay technique. The time variations in the different indices are clearly correlated on short time scales.

\section{VARIATIONS OF THE ASYMMETRY IN VARIOUS SOLAR-ACTIVITY INDICES ON SHORT AND LONG TIME SCALES}

We determined the asymmetry indices in the standard way, $A=(N-S) /(N+S)$, where $N$ and $S$ are the corresponding activity indices for the northern and southern hemispheres. For our particular investigation, we needed the mean asymmetries for specific intervals of time and latitude. Since $A$ depends nonlinearly on $N$ and $S$, we must define the adopted procedure for averaging $A$. When deriving the mean asymmetries, we first found the monthly average (for the green-line brightness and net magnetic flux) or monthly summed (for areas or sunspot numbers) solar-activity indices for the chosen latitude zone in the northern and southern hemispheres separately, then calculated $A$ according to the above formula. In some cases, we also smoothed the resulting asymmetry values; this will always be specially indicated in the text.

Normally, the N-S asymmetry refers to the difference between the entire northern and southern hemispheres. However, as is well known, the solar activity exhibits a zonal structure. For this reason, we found it expedient to use a latitude-dependent activity index. In doing so, we took $N$ and $S$ to be the values of a given index for solar latitude zones placed symmetrically about the equator.

Figure 1 shows latitude-time diagrams for the asymmetries in three of the activity indices considered the net magnetic flux, coronal green-line brightness, and the total sunspot area. The long-term, largescale asymmetry variations are shown here. The upper two diagrams were constructed using the semiannual mean asymmetry values for 10-degree latitude zones. The lowest panel was constructed by calculating the semiannual mean asymmetries for the total sunspot numbers in the three latitude zones $0^{\circ}-10^{\circ}$, $10^{\circ}-20^{\circ}$, and $>20^{\circ}$, then averaging using a ninthorder polynomial using the Savitzky-Golay method (see http://www.mathworks.com). This method suppresses noise but preserves high-frequency components. This averaging was needed to reveal largescale variations in the asymmetry, since the asymmetries of areas and sunspot numbers undergo broader variations than the asymmetries of the other indices (for example, $A$ can reach \pm 1 in periods when there are no sunspots in one hemisphere); thus, the map would be fairly "noisy" without such averaging.

Figure 1 demonstrates that the asymmetries in these indices vary similarly over the entire latitude range (although the $A$ values themselves are different). This can be seen from the alternation of dark and light strips, which is similar in all three diagrams in Fig. 1. Note that the asymmetry in the 

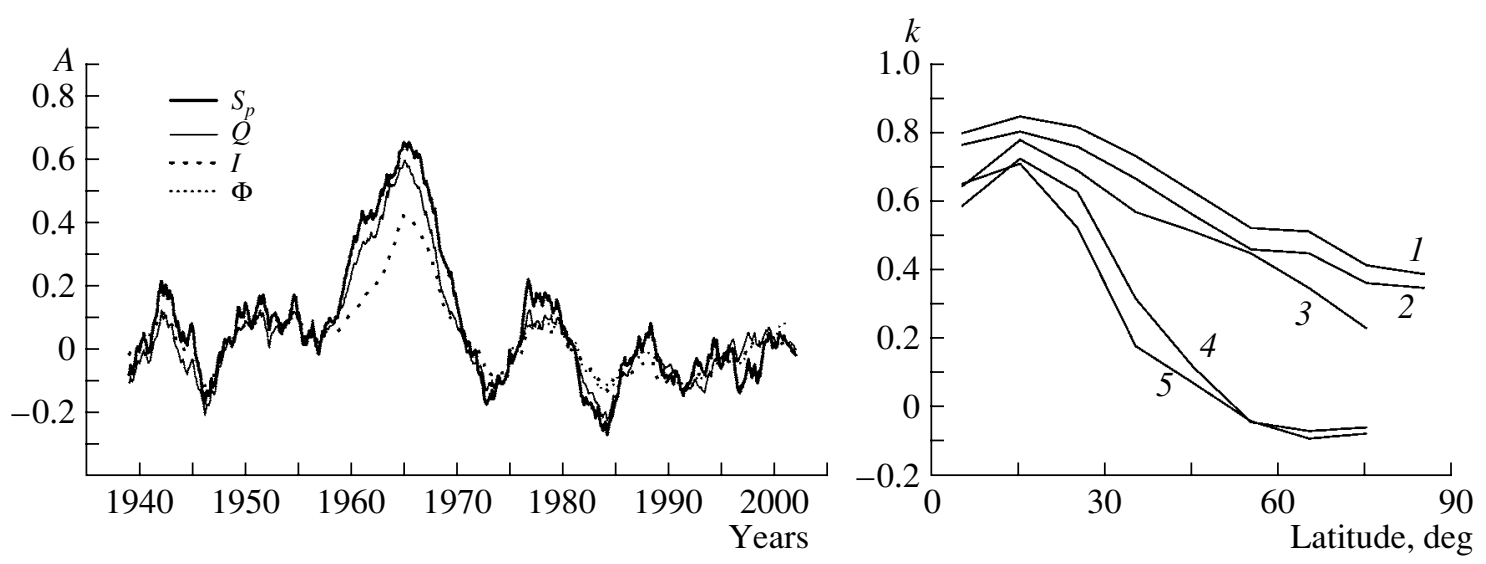

Fig. 3. Left: asymmetries in the four activity indices at latitudes $0^{\circ}-30^{\circ}$ subjected to a running-average with a 49 month window. A similarity can be noted in the asymmetry variations on long time scales. Right: correlations between the asymmetries for various pairs of solar-activity indices: $(1)$ the green-line brightness $I$ and the total sunspot number $Q$ in 10-degree latitude zones, (2) $I$ and the total sunspot area $S_{p}$, (3) $I$ and the magnetic flux $\Phi$ in 10-degree zones, (4) $\Phi$ and $Q$, and (5) $\Phi$ and $S_{p}$.

total sunspot number (which is not presented here) is virtually identical to the asymmetry in the sunspot areas (shown in the lowest diagram). During the first half of the time interval considered, the corona was generally brighter in the northern than in the southern hemisphere. The opposite was true during the second half of the interval, although the asymmetry was considerably less pronounced. In other words, positive asymmetry (with the northern hemisphere exceeding the southern hemisphere in brightness) was predominant in 1939-2001. A substantial increase in the asymmetry was observed in 1965-1968, as has repeatedly been noted before [11]. The vertical strips in the latitude-time diagram result from nearly simultaneous changes in the asymmetry at all latitudes. We demonstrated this effect previously for the time variation of the $\mathrm{N}-\mathrm{S}$ asymmetry of the green-line emission in 10-degree latitude zones [18, 19].

We also considered another sunspot-activity index: the traditional Wolf numbers [19, Fig. 3]. The cyclic variations in the sunspot number, green-line brightness, and sunspot area were considered for the northern and southern hemispheres separately. The asymmetry indices for these three parameters increase or decrease nearly simultaneously, and their variations correlate well in terms of power on both long and short time intervals. All three parameters demonstrate that the northern hemisphere dominated during the first half of the considered time interval and the southern hemisphere during the second half. This provides evidence for a long-period wave with a period of about 40 years in the variations, as has already been mentioned in some previous studies [1,7].

Figure 2 shows the variations in the asymmetries calculated from the four activity indices averaged over the zone of spot formation, $0^{\circ}-30^{\circ}$. This figure was constructed by first calculating monthly mean $A$ values for all the indices, then smoothing them using the Savitzky-Golay technique with a 13th-order polynomial. Figure 2 shows that the resulting curves are very similar, even in specific small details. For example, an asymmetry increase in 1965-1968, a decrease near 1983, and a sharp asymmetry outburst in 1986 are clearly visible in all the indices. Table 1 presents the pair-correlation coefficients for the asymmetries in the indices; $k_{A}(X, Y)$ denotes the correlation coefficient for the asymmetries in indices $X$ and $Y$.

Figure 3 (left) shows the same asymmetry indices subjected to a running-averaging procedure with a 49 month window. In contrast to Fig. 2, the highfrequency component is removed, leaving only the slowly varying component. We can see from Fig. 3 that the asymmetry curves are likewise similar in all their large-scale features. This similarity is reflected by the very high correlation coefficients for pairs of indices: $k_{A}\left(S_{p}, I\right)=0.950, k_{A}(Q, I)=0.960$, $k_{A}\left(S_{p}, Q\right)=0.975$. The presence of several maxima and minima in the left-hand graph in Fig. 3 suggests the existence of a quasiperiodic wave with a period of $\sim 12 \mathrm{yr}$. It is important to realize that, although this smoothing procedure reveals the $11-y r$ cyclic variations in the activity indices themselves, these variations do not display a correlation as high as that for the asymmetry. In particular, while $k_{A}\left(S_{p}, I\right)=$ 0.95 , the correlation coefficients for the smoothed indices themselves taken separately for the northern and southern hemispheres are 0.76 .

The right-hand graph in Fig. 3 represents the paircorrelation coefficients for the asymmetries in the four indices as functions of latitude. The green-corona brightness and magnetic flux are taken for 10-degree latitude zones, while the area and total number of 


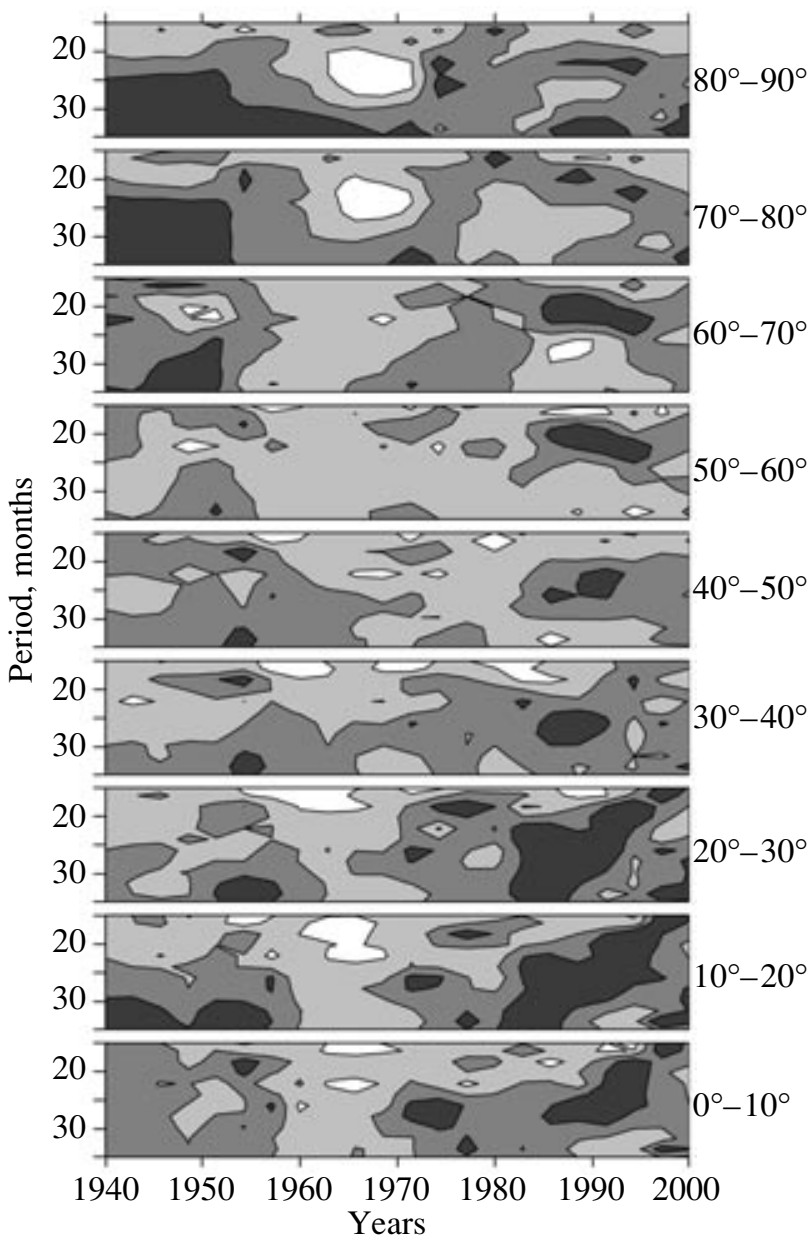

Fig. 4. SVAN diagrams for the asymmetry of the coronal green-line brightness in 10-degree latitude zones. The contour increment is 0.065 , with black corresponding to amplitudes exceeding 0.195 .

sunspots are calculated for the entire spot-formation zone. The semiannual mean asymmetry values are used. The $A$ values for the coronal green-line brightness and the total sunspot number show the best correlation in all latitude zones; the correlation between the $A$ values for the green-corona brightness and the total sunspot area is slightly lower, again in all latitude zones. The highest correlation of the asymmetries is observed at latitudes of $10^{\circ}-20^{\circ}$ for all the pairs of activity indices; the correlation is considerably lower in the polar region. At the same time, note the following interesting and not fully understood fact: the asymmetry in the green corona at middle latitudes above $30^{\circ}$, where virtually no sunspots emerge, remains well correlated with the asymmetry in the sunspot areas, which is mainly contributed by latitudes below $30^{\circ}$. Note also that the magnetic flux in the polar zone exhibits a negative, although weak, correlation with the total number and area of sunspots. This may suggest that the magnetic flux in

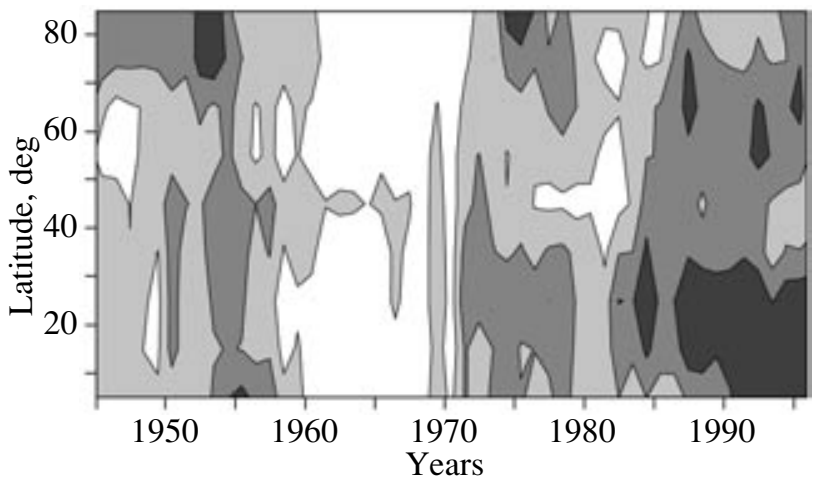

Fig. 5. Time and latitude distributions of the sum of the squared amplitudes of oscillations in the green-line asymmetry. The contour increment is 0.04 , with black corresponding to values in excess of 0.12 .

the polar zone is predominantly controlled by largescale fields, while the green-corona brightness is substantially affected by local fields at all latitudes. It should, however, be kept in mind that we calculated the magnetic flux using observations of the line-ofsight magnetic field, which is essentially tangential at high latitudes.

Thus, the increases and decreases in $A$ for all four activity indices here are essentially simultaneous and correlate well in magnitude on both long and short time intervals.

Our analysis in this section demonstrates that the asymmetry represents a general phenomenon in the Sun, which is manifest simultaneously and in nearly the same way in different indices. It is this property that substantially distinguishes the asymmetry from the activity indices themselves. We find evidence that the asymmetry is a fundamental characteristic of solar activity, which determines the degree of coincidence between the processes of magnetic-field generation in the two hemispheres.

\section{QUASIBIENNIAL OSCILLATIONS IN THE N-S ASYMMETRIES OF VARIOUS INDICES}

The QBOs are among the most interesting periodic oscillations observed in the solar activity. There have been fairly numerous studies devoted to the QBOs in recent years (see references in [20]). It has even been suggested that the QBO phenomenon is no less important than the 11-year solar cycle. There are grounds to believe that the QBOs are related to processes near the base of the convection zone with similar periodicities. Obridko and Gaziev [21] have shown that QBOs are clearly manifest in the asymmetry of magnetic fields inferred from $\mathrm{H} \alpha$ data. Oscillations in this frequency range were also detected by Knaack et al. [4]. 


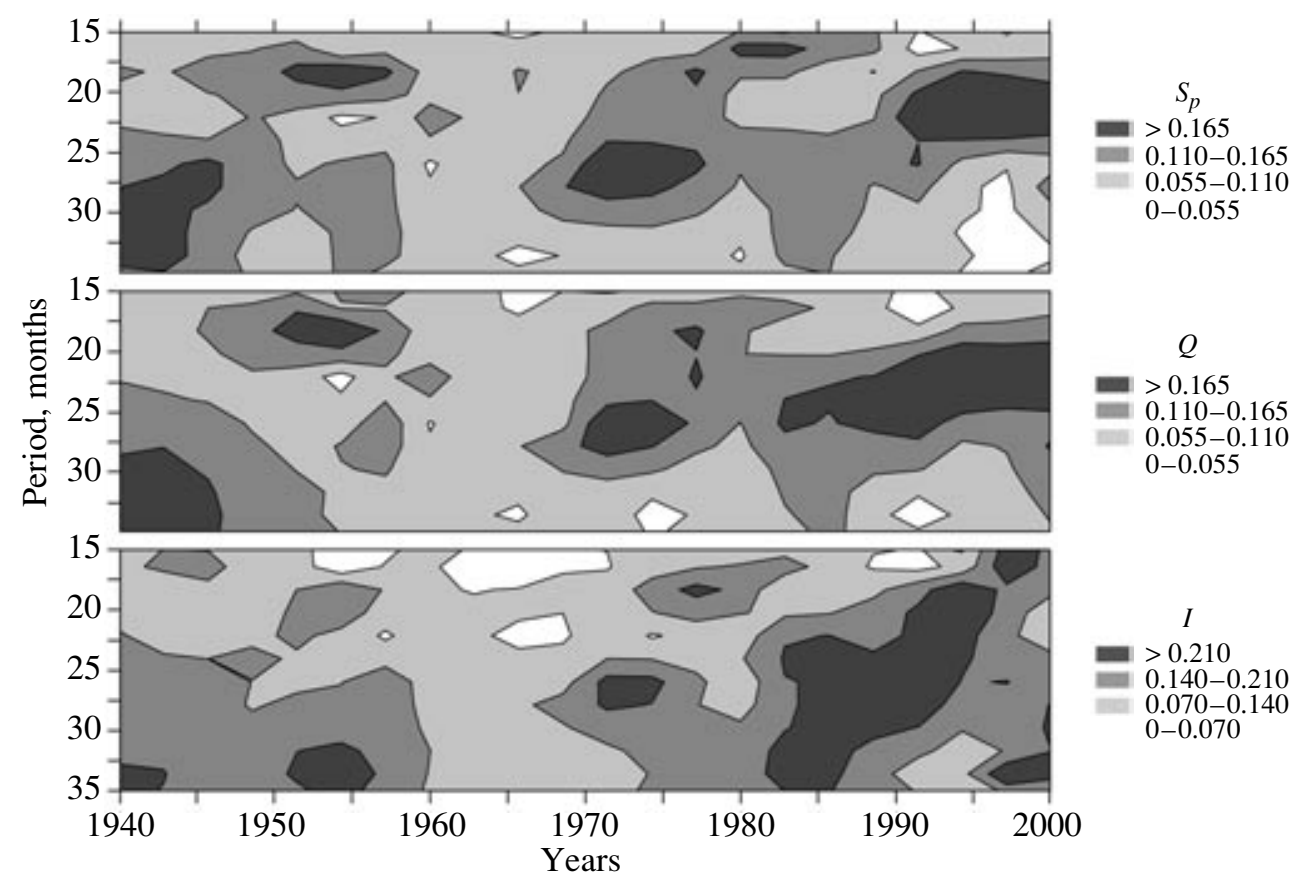

Fig. 6. SVAN diagrams for the asymmetries in the sunspot area, total sunspot number, and green-corona brightness over the spot-formation zone, $0^{\circ}-30^{\circ}$.

The time variations shown in Fig. 2 indicate that short-period oscillations are present in the asymmetry variations. Even without a more detailed analysis, we can see that the characteristic time scale of these oscillations is about 1.5-3 yr. A Fourier analysis testifies to the presence of quasibiennial variations in the spectrum of the asymmetry. Consideration of oscillations with periods ranging from 15 months to $4 \mathrm{yr}$ for three latitude zones indicated that the oscillations in the spot-formation zone, $0^{\circ}-30^{\circ}$, are enhanced in the period range 2.2-2.5 yr. At high latitudes, $60^{\circ}-90^{\circ}$, two peaks can be identified, near 2.5 and 3 yr. At middle latitudes, $35^{\circ}-55^{\circ}$, the oscillations do not exhibit well-pronounced maxima, suggesting that, if QBOs are present in this latitude zone, they are much weaker than at lower and higher latitudes.

We used a spectral-variation analysis (SVAN) to study the time and latitude dependences of the QBOs. We employed an original SVAN code, which, in contrast to widely used codes described in the literature (see, e.g., [23]), includes normalization to a standard value (division by the standard deviation). This normalization reduces all oscillations to a single scale, with the sum of the squared amplitudes being unity in any spectrum. This enables us to compare the SVAN results for processes that are described quantitatively using different units.

A SVAN consists of successive Fourier decompositions over running time intervals. A Fourier decomposition is calculated for a time window of a chosen length, after which this window is shifted by some time interval and the entire procedure repeated. The resulting expansions are then used to construct a general map of the oscillation amplitudes (SVAN diagram) in time-(oscillation period) coordinates. We used the mean monthly asymmetries for our analysis, with a running window of 132 months and a time shift of 12 months. Normalization to the standard deviation was done in each window. We determined the amplitudes of oscillations with periods of 6-44 months. Although the relative power of any oscillation is characterized by its squared amplitude, analyzing the amplitudes themselves is more convenient in some cases. Obviously, such a representation of the results cannot affect the conclusions. We will always specify below which quantity is presented in figures and tables.

Figure 4 shows SVAN diagrams (oscillation amplitudes) for the asymmetry in the coronal green-line brightness in 10-degree latitude zones. The entire range of variations in the amplitude of the asymmetry oscillations is divided into four gradations. Darker regions correspond to larger amplitudes, with black used for amplitudes exceeding 0.195 (recall that the sum of the squared amplitudes over all periods in any running window is unity). Figure 4 demonstrates the presence of quasibiennial oscillations (periods of 20-35 months) over most of the time period studied. QBOs are especially pronounced in the equatorial zone after 1970. Longer period harmonics are manifest at high latitudes, especially in the 1940s 


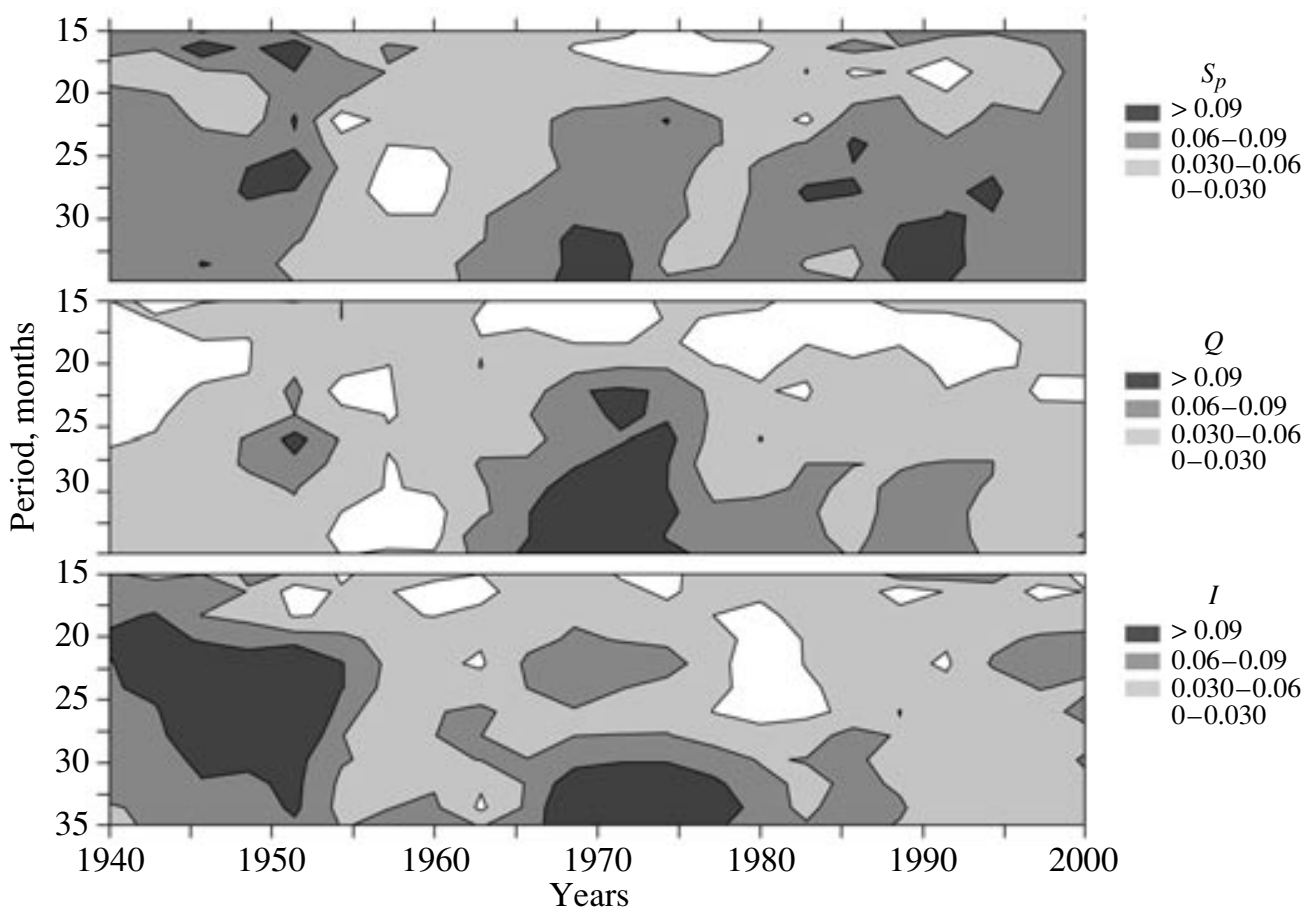

Fig. 7. Same as Fig. 6 for the indices $S_{p}, Q$, and $I$ themselves.

and 1950s. On the other hand, the QBOs are always weak at latitudes of $50^{\circ}-60^{\circ}$, and QBOs were virtually absent at all latitudes in the 1960s. Note that the asymmetry was very strong precisely during this period.

Figure 5 shows maps of the time and latitude distributions of the squared amplitudes of the QBOs observed in the asymmetry of the coronal green-line brightness. We summed the squared amplitudes for three oscillation periods that roughly correspond to QBOs (18.86, 22.0, and 26.4 months). Darker areas correspond to higher amplitudes, with black denoting values exceeding 0.12 . We can clearly see that (a) the QBOs are enhanced during the second half of the time interval studied (after 1970), (b) the QBOs are more pronounced at low than at high and, even more so, than at middle latitudes, and (c) we can identify several vertical strips indicating that the QBOs in the asymmetry are enhanced or weakened at all latitudes

Table 2. Correlation coefficients for the sums of the squared QBO amplitudes of the asymmetry and the activity indices themselves

\begin{tabular}{l|c|c}
\hline Indices & $k_{A s}(X, Y)$ & $k_{s}(X, Y)$ \\
\hline$I-S_{p}$ & $0.693 \pm 0.099$ & $0.50 \pm 0.20$ \\
$I-Q$ & $0.854 \pm 0.071$ & $0.29 \pm 0.13$ \\
$S_{p}-Q$ & $0.889 \pm 0.063$ & $0.56 \pm 0.11$ \\
\hline
\end{tabular}

during certain time intervals. A comparison of Figs. 5 and 1 reveals a general large-scale anticorrelation between the asymmetry and the power of its QBOs. This point will be considered in more detail in Section 5 .

We carried out a wavelet analysis of the asymmetry in the coronal green-line brightness, again for 10-degree latitude zones [22, Fig. 5]. The wavelet diagrams provide higher time resolutions than the SVAN diagrams, but at the expense of spectral resolution. A gradual drift of the QBOs from the equatorial zone to high latitudes was observed during 1943-1948 (the growth phase of cycle 18) and 1984-1993 (the growth and maximum phases of cycle 22); this may somehow be related to the presence of the abovementioned $\sim 40$ yr wave in the asymmetry variation. In other cycles, QBOs were present during some time intervals over a fairly broad range of latitudes without any pronounced drift (for example, QBOs were clearly visible in $1973-1974$ at latitudes of $0^{\circ}-40^{\circ}$ ). As in the SVAN diagrams, there is a decrease in the $\mathrm{QBO}$ power during the 1960s.

Quasibiennial oscillations are also clearly pronounced in the SVAN and wavelet diagrams for the magnetic flux. At low and middle latitudes, they are most intense near 1980 and 1995. By and large, the characteristic enhancements of the QBOs in the magnetic-flux asymmetry over the spot-formation zone, $0^{\circ}-30^{\circ}$, agree in time with the enhancements revealed in the other indices studied. A 4-6-year 
Table 3. Sums of the squared oscillation amplitudes for various frequency ranges

\begin{tabular}{c|c|c|c|c|c}
\hline \multirow{2}{*}{ Range } & \multirow{2}{*}{ Periods, months } & \multicolumn{2}{|c|}{ Asymmetry } & \multicolumn{2}{c}{ Indices } \\
\cline { 3 - 6 } & & $S_{p}$ & $I$ & $S_{p}$ & $I$ \\
\hline I & $25.2-12.6$ & 0.06000 & 0.06278 & 0.01988 & 0.01606 \\
II & $18.9-37.8$ & 0.06016 & 0.07270 & 0.01279 & 0.01517 \\
III & $94.5-189.0$ & 0.03971 & 0.08942 & 0.32392 & 0.36761 \\
\hline
\end{tabular}

( $\sim 1500$ day) period can also be identified in the wavelet diagrams, which is especially pronounced in the magnetic-flux values near the maximum of cycle 22 in 1990 [19, Fig. 5].

Figure 6 presents SVAN diagrams for the asymmetry of the indices $S_{p}, Q$, and $I$ at latitudes of $0^{\circ}-30^{\circ}$. The features detected in the asymmetry of the green-line brightness can also be seen in the asymmetry of these other activity indices. In particular, the decrease in the QBO amplitude during the 1960 s and the substantial increase during and after the 1970 s can be traced in the asymmetries of the sunspot area $S_{p}$ and the total sunspot number $Q$. There are also other periods of $\mathrm{QBO}$ enhancements in the spot-formation zone. Of course, the similarity of the SVAN diagrams for the asymmetries in $S_{p}$ and $Q$ is quite natural, although, as is noted above, these indices are not equivalent, and the parameters relating them are time-dependent [24]. At the same time, the SVAN diagram for the green corona also exhibits a general similarity with the other two SVAN diagrams, although complete agreement could obviously hardly be expected in this case.

In addition to the SVAN diagrams for the activityindex asymmetries at latitudes of $0^{\circ}-30^{\circ}$, we constructed SVAN diagrams for the indices themselves, the total sunspot area $S_{p}$, total sunspot number $Q$, and the green-corona brightness $I$ (Fig. 7). First and foremost, we note that the SVAN diagrams for these three solar-activity indices are considerably less similar than the the SVAN diagrams for the asymmetries in these same indices (cf. Fig. 6). However, the most important difference is that, unlike Fig. 6, these diagrams do not reveal pronounced QBOs in the activity indices themselves. A comparison of Figs. 6 and 7 indicates that the QBOs are much more clearly manifest in the asymmetries than in the solar-activity indices themselves. In addition, the entire range of variation of the oscillation amplitudes, indicated on the right in Figs. 6 and 7, is a factor of 1.5-2 smaller for the indices than for their asymmetries.

Table 2 compares the correlation coefficients for the sums of the three squared amplitudes in the QBO range (periods of 18.86, 22.0, and 26.4 months).
A pair-wise comparison is presented for the correlation coefficients of the sums of the squared QBO amplitudes in the spot-formation zone, $0^{\circ}-30^{\circ}$, obtained for the asymmetry, $k_{A s}(X, Y)$, and for the indices themselves, $k_{s}(X, Y)$.

We can see from Table 2 that (i) the paircorrelation coefficients for the asymmetry are statistically significant, while the correlation coefficients for the indices exceed $3 \sigma$ only in the last row, and (ii) the correlation coefficients for the asymmetry are definitely higher than for the activity indices themselves. This is especially clear when comparing the asymmetries of the coronal green-line brightness and the total sunspot number.

It has been repeatedly noted in the literature that oscillations observed on the Sun include those with characteristic time scales of one to two years, QBOs, and the 11-year cycle. The relative contributions of oscillations within these ranges to the overall spectrum for oscillations from 1 month to $63 \mathrm{yr}$ is of interest. We calculated the sums of the squared amplitudes in the spot-formation zone for various frequency ranges in the Fourier spectrum (recall again that the sum of the squared amplitudes of all oscillations is unity), then isolated intervals in the observed spectrum corresponding to oscillations with periods of about $1-2 \mathrm{yr}$ (range I), 1.5-3 yr (range II), and $8-16$ yr (range III) (Table 3 ).

The 11-year cycle is predominant in the activity indices. The oscillation power in range III exceeds $30 \%$ of the total power of all the oscillations in the spectrum. At the same time, the oscillations in the quasibiennial (II) and high-frequency (I) ranges are a factor of 20-30 weaker. The situation changes radically when we consider the asymmetry spectrum. In range III, the relative power of oscillations in the asymmetry is a factor of $5-10$ lower than the power of the oscillations in the activity indices. On the other hand, the oscillations with periods of $1.5-3 \mathrm{yr}$ and $1-2$ yr are enhanced by a factor of $3-5$, and become comparably significant to the roughly 11 -year oscillations. 


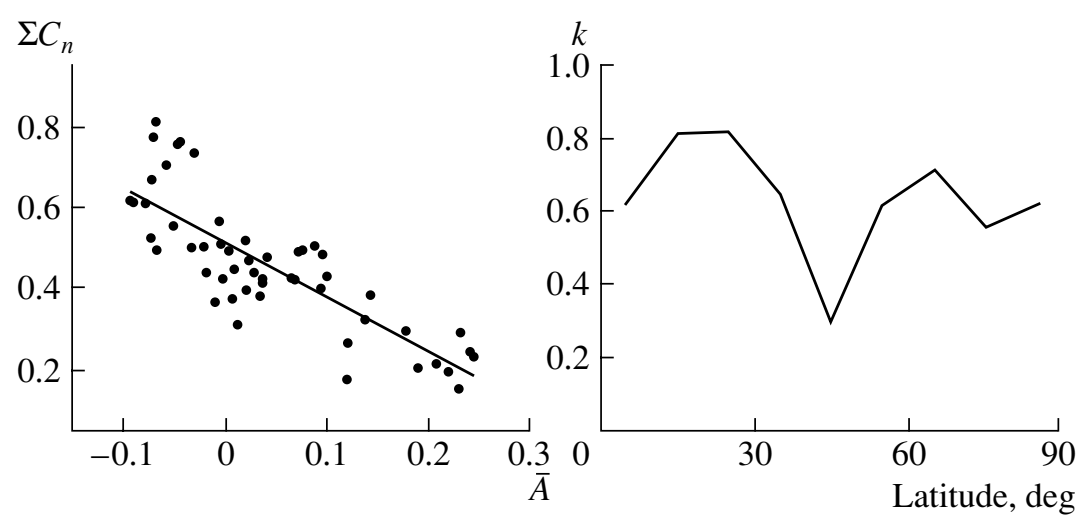

Fig. 8. Left: relationship between the sum of the squared amplitudes of oscillations with periods of $18.8-26.4$ months and the mean asymmetry in a running window for latitudes $0^{\circ}-30^{\circ}$. The correlation coefficient is 0.82 . Right: latitude dependence of the correlation coefficient characterizing the relation between the sum of the squared QBO amplitudes and the amplitude of the asymmetry.

\section{RELATION BETWEEN THE ASYMMETRY AND ITS QUASIBIENNIAL OSCILLATIONS}

A comparison of Figs. 5 and 1 suggests the presence of a general large-scale anticorrelation between the asymmetry and the power of its QBOs. This is also supported by other tendencies that can be distinguished in these figures. In particular, the interval of weakened QBOs during the 1960s coincides in time with an increase in the asymmetries in the greencorona brightness and the areas and total number of sunspots. We conclude that a negative correlation between the QBO power and the asymmetry amplitude $A$ can be traced over the entire time interval and over all latitudes.

The left-hand graph in Fig. 8 shows the relationship between the sum of the amplitudes of the QBOs observed in the green-line brightness asymmetry for latitudes $0^{\circ}-30^{\circ}$ and with periods from 18.8 to 26.4 months and the mean asymmetry in the running window of the SVAN analysis. The correlation coefficient for the negative correlation between these quantities at these latitudes is $0.82 \pm 0.05$.

The negative correlation between the relative sum of the QBO amplitudes and the asymmetry magnitude can also be traced in all other latitude zones. The latitude dependence of the correlation coefficient is shown in Fig. 8 (right). The highest correlation coefficients occur at $10^{\circ}-20^{\circ}$ and $60^{\circ}-70^{\circ}$. These two regions are separated by a narrow zone $\left(40^{\circ}-50^{\circ}\right)$ where the correlation coefficient is very low. Note that the asymmetry in the green line reaches its maximum in 1965-1968 precisely near this zone (Fig. 1). Furthermore, high-frequency oscillations disappear almost completely in this zone. Note as well that the boundary delimiting the regions of low-latitude and polar magnetic fields is located in this same zone.
The fact that the relationship between the relative QBO power and the asymmetry amplitude typified by the left-hand graph in Fig. 8 holds even in regions where the southern hemisphere dominates (i.e., the asymmetry is negative) appears somewhat strange. It would be more natural to see a similar relationship between the absolute value of $A$ and the QBO power. Since there were virtually no intervals with large negative asymmetries during 1939-2001, we continued our analysis of this problem using a long series of data on the areas and total numbers of sunspots covering the period 1874-2002 [24]. We have shown that precisely the absolute value of the asymmetry for the index $A$ is important, and that the $\mathrm{QBO}$ power also decreases with increase in this quantity, in regions of both negative and positive $A$ values [24, Fig. 4]. Unfortunately, Figs. 2 and 3 in [24], which are similar to the upper two graphs in Figs. 6 and 7 here but refer to the longer period of $1874-2002$, were spoiled in printing and cannot be used.

\section{CONCLUSION}

We have analyzed the north-south asymmetry $A$ using four different indices characterizing the activity at various levels of the solar atmosphere: the brightness of the coronal green line, the total sunspot area, the total sunspot number, and the net magnetic flux. Our analysis demonstrates that the $\mathrm{N}-\mathrm{S}$ asymmetry is a fundamental characteristic of the solar activity, which displays its own trends and is not directly controlled by the cyclic behavior of the solar activity. Thus, the $\mathrm{N}-\mathrm{S}$ asymmetry is a specific, independent and very promising tool for analyses of solar-activity variations.

We have obtained the following main results.

(1) Similar behavior is visible in the time variations (Fig. 2; Fig. 3, left) of the N-S asymmetry of 
all the solar-activity indices considered in the spotformation zone, $0^{\circ}-30^{\circ}$, on both short $(1.5-3 \mathrm{yr})$ and long $(\sim 12 \mathrm{yr})$ time scales. The northern hemisphere dominates during the first half of the time interval studied (with a well-pronounced maximum in 1964-1966), and the southern hemisphere in the second half.

We emphasize that the solar-activity indices we have considered correspond to completely different solar phenomena associated with completely different mechanisms for the interaction between the magnetic field and matter. In one case, the magnetic field controls the convective motions in subphotospheric layers (sunspots), while, in another, the magnetic field controls the flux of magnetoacoustic waves (coronalheating mechanism). Nevertheless, comparisons between the results for different formations on the Sun testify to a surprising similarity in their asymmetries.

(2) We also calculated the quantity $A$ separately for narrower latitude zones, and considered correlations between pairs of asymmetry values for various activity indices in these zones. The highest correlation coefficient for any pair is reached at latitudes of $10^{\circ}-20^{\circ}$ (Fig. 3, right). The asymmetry in the greencorona brightness correlates well with the asymmetries in the total sunspot number and the sunspot area. This correlation remains significant at high latitudes, where sunspots are not observed. We conclude that the green-corona-brightness asymmetry is determined predominantly by a single parameter, which is related to the local magnetic fields. Conversely, the magnetic-flux asymmetry is determined by two magnetic-field components, as is reflected by the sign reversal in the corresponding curves for high latitudes in the right-hand graph of Fig. 3. It seems most reasonable to suppose that these components correspond to the local low-latitude fields and largescale polar fields.

The high correlation of the high-latitude coronal brightness with the sunspot numbers may not by itself imply a direct physical relationship between these two phenomena. Many solar processes give the impression that the rhythm of the process is more important than direct energetic control. For example, in our case, the sunspot number may reflect the overall rhythm of the associated solar processes, which governs both the local magnetic fields and the coronal-brightness variations. Since the asymmetry is a difference quantity, it is potentially a sensitive tool for recognizing this overall rhythm.

(3) Quasibiennial oscillations (QBOs) were detected in the asymmetries of all the activity indices considered. We studied these QBOs using an original code for spectral-variation analysis (SVAN), which includes normalization to a standard value. We constructed SVAN diagrams for the green line and magnetic flux in 10-degree latitude zones, which demonstrate the existence of long periods in which there is a steady enhancement or weakening of the QBO power that is virtually simultaneous over a broad range of latitudes (Fig. 4).

(4) The SVAN diagrams for the spot-formation zone, $0^{\circ}-30^{\circ}$, show that specific features of the quasibiennial variations displayed in the asymmetry of the green-line brightness can also be traced in the behavior of the other activity indices. Similar enhancements can be noted in the QBOs, and coincide in both time and frequency (Fig. 6). For example, a decrease in the QBO amplitude can be observed in the asymmetries of the sunspot areas and total numbers during the mid-1960s, followed by an appreciable increase during and after the 1970s. QBOs are continuously present in the interval 1970-2000. The correlation coefficients for the correlations between the sums of the squared QBO amplitudes for these three indices reach $\sim 0.7-0.9$. At the same time, the $\mathrm{QBOs}$ are not oscillations with a steady period but are instead a combination of pulses with a period of $1.5-3 \mathrm{yr}$.

(5) We carried out a spectral-variation analysis for the activity indices themselves and compared the results with the SVAN data for the asymmetries. The QBOs are much more pronounced in the N-S asymmetries than in the corresponding activity indices. The amplitudes of the QBOs in the indices are much more weakly correlated with one another than are the amplitudes of the QBOs in the asymmetries: the correlation coefficients for correlations between the $\mathrm{QBO}$ amplitudes are as low as $\sim 0.3-0.55$ in this case.

(6) We have discovered the unexpected effect that the manifestation of QBOs in the asymmetries of the studied parameters of solar activity, especially the green-corona brightness, appears to be in antiphase with the asymmetry itself. For example, the substantial increase in the asymmetry of all the activity indices in the mid-1960s is accompanied by an appreciable decrease in the QBO amplitude in all our SVAN diagrams. The negative correlation coefficient for the negative correlation between the sum of the three QBO amplitudes and the green-line asymmetry at latitudes of $0^{\circ}-30^{\circ}$ is $0.82 \pm 0.05$.

On the whole, the nature of the asymmetry of the solar indices remains unclear. As a rule, the most widespread dynamo theories do not include this asymmetry, instead assuming that both solar hemispheres are identical. The asymmetry could be the manifestation of a slowly varying relic magnetic field, but its existence has not been proven in any way, and is doubtful from the theoretical standpoint. To account for the asymmetry, the relic magnetic field should have fairly unusual properties: it should 
not obey the Hale polarity law and must have a pronounced asymmetric component. A sphericalharmonic expansion of the large-scale magnetic field yields a coefficient that formally corresponds to a quasimonopole component. However, the nature of this effect is not understood, and it is quite possible that this coefficient results from observational errors (see, e.g., [25]).

We have the impression that the solar activity is generated largely independently in the two hemispheres, being governed by the differential rotation and meridional flows in each. However, since the cyclic variations in the two hemispheres exhibit a general temporal and energetic similarity, we suggest that some currently unknown mechanism determines the degree of similarity of the activity-generating processes in the two hemispheres. This mechanism should be superposed on and independent of the basic generation mechanism. The north-south asymmetry is apparently a quantitative measure of the properties of this mechanism. We should recall again in this context that the degree of similarity between the time variations and the manifestation of quasibiennial oscillations in the asymmetries of various indices is higher than in the original activity indices themselves. Thus, the asymmetry remains an unresolved problem in the question of the origin of the solar magnetic field.

\section{ACKNOWLEDGMENTS}

This work was supported by the Russian Foundation for Basic Research (project no. 05-02-16080) and VEGA grant no. 2/4013/24 of the Slovak Academy of Sciences. The NSO/Kitt Peak data used here were jointly prepared by the NSF/NOAO, NASA/GSFC, and NOAA/SEL.

\section{REFERENCES}

1. M. Carbonell, R. Oliver, and J. L. Ballester, Astron. Astrophys. 274, 497 (1993).

2. M. Waldmeier, Sol. Phys. 29, 332 (1971).

3. K. J. Li, J. X. Wang, S. Y. Xiong, et al., Astron. Astrophys. 383, 648 (2002).

4. R. Knaack, J. O. Stenflo, and S. V. Berdyugina, Astron. Astrophys. Lett. (2005) (in press).

5. G. Vizoso and J. L. Ballester, Astron. Astrophys. 229, $540(1990)$.

6. J. Mariş, M. D. Popescu, and M. Mierla, Rom. Astron. J. 12 (2), 131 (2002).
7. Yu. A. Nagovitsyn, Izv. Gl. Astron. Obs. Pulkovo, No. 212, 145 (1998).

8. P. I. Duchlev, Sol. Phys. 199, 211 (2001).

9. Yu. I. Vitinskii, M. Kopetskii, and G. V. Kuklin, Statistics of Spot-Formation Activity of the Sun (Nauka, Moscow, 1986) [in Russian].

10. H. W. Newton and A. S. Milson, Mon. Not. R. Astron. Soc. 115, 398 (1955).

11. J. Sýkora, Solar and Interplanetary Dynamics, Ed. by M. Dryer and E. Tandberg-Hanssen (Reidel, Dordrecht, 1980), p. 87.

12. V. Rušin, Bull. Astron. Inst. Czech. 31, 9 (1980).

13. J. Sýkora, Bull. Astron. Inst. Czech. 22, 12 (1971).

14. J. Sýkora, Contrib. Astron. Obs. Skalnaté Pleso 22, 55 (1992).

15. J. Sýkora, Adv. Space Res. 14 (4), 73 (1994).

16. O. G. Badalyan, V. N. Obridko, and J. Sýkora, Sol. Phys. 199, 421 (2001).

17. J. Sýkora and J. Rybák, Adv. Space Res. (2004) (in press).

18. J. Sýkora, O. G. Badalyan, and M. Storini, Adv. Space Res. 29 (12), 1975 (2002).

19. O. G. Badalyan, V. N. Obridko, J. Rybák, and J. Sýkora, in Proceedings of the Second Solar Cycle and Space Weather Euroconference, Vico Equense, Italy, 2001, Ed. by H. Sawaya-Lacoste (ESA Publications Division, Noordwijk, 2002), ESA SP-477, p. 201.

20. V. N. Obridko and B. D. Shel'ting, Astron. Zh. 78, 1146 (2001) [Astron. Rep. 45, 1012 (2001)].

21. V. N. Obridko and G. Gaziev, in The Solar Cycle. Proceedings of the National Solar Observatory/Sacramento Peak 12th Summer Workshop, Ed. by K. L. Harvey (Astron. Soc. Pac., San Francisco, 1992); Astron. Soc. Pac. Conf. Ser. 27, 410 (1992).

22. O. G. Badalyan, V. N. Obridko, J. Rybák, and J. Sýkora, in Solar Variability as an Input to the Earth's Environment, Ed. by A. Wilson (ESA Publications Division, Noordwijk, 2003), ESA SP-535, p. 63.

23. A. Dzewonski, S. Block, and M. Landisman, Bull. Seismol. Soc. Am. 59, 427 (1969).

24. O. G. Badalyan and V. N. Obridko, in Climatic and Ecological Aspects of Solar Activity, Ed. by V. I. Makarov and V. N. Obridko (Gl. Astron. Obs. Ross. Akad. Nauk, St. Petersburg, 2003), p. 33.

25. J. T. Hoeksema and P. H. Scherrer, The Solar Magnetic Field-1976 through 1985, WDCA Report UAG-94 (NGDC, Boulder, 1986).

\section{Translated by A. Getling}

